\title{
Herniation of mucosal epithelium into the submucosa in chronic ulcerative colitis
}

\author{
JENNIFER L. DYSON \\ From the City Hospital, Nottingham
}

SYNOPSIS Herniation of the glandular epithelium into the submucosa has been observed in 11 out of 27 cases of chronic ulcerative colitis. Glandular herniation was associated with thickening of the muscularis mucosae, with interruption of the muscularis mucosae by lymphoid follicles, and, in five of the 11 cases, with significant crowding of the glands of the mucosa.

This study strongly suggests that sustained contraction of the muscularis mucosae, which has been shown by others to be a major feature of chronic ulcerative colitis, is the prime factor in the formation of downgrowths or herniations of the glandular epithelium into the submucosa.

Comparison of the cases in which cancer developed with those where there was glandular herniation led to the conclusion that they are independent associations of chronic ulcerative colitis, and that glandular herniation plays no part in the development of dysplasia or cancer.

Chronic ulcerative colitis produces shortening of colon (Bargen and Weber, 1931), mucosal atrophy (Morson, 1968), and thickening of the muscularis mucosae (Goulston and McGovern, 1969; Morson, 1969). Misplaced epithelium in the submucosa, first described by Dukes (1954), is rarely commented upon. It may not be obvious in the acute phase of the disease, when the formation of deep abscesses may destroy the epithelium. But greater recognition of the long-term risks of carcinoma developing in ulcerative colitis (Hinton, 1966; Watts, De Dombal, Watkinson, and Goligher, 1966) has led to increased prophylactic surgery, especially among those at risk (the young, those with total colitis, and those with disease of more than 10 years' duration), and this makes it easier for the pathologist to evaluate the histological changes in chronic ulcerative colitis without the destructive overlay of acute inflammation.

This study considers the mechanism by which glandular herniation develops, and its relationship to carcinoma.

\section{Methods}

This survey was carried out as part of a review of patients with chronic ulcerative colitis at the Central Middlesex Hospital, London, and at the City Hospital, Nottingham. All had had total colitis for a Received for publication 28 October 1974. minimum of three years and had been treated by total colectomy or panproctocolectomy, the operations at the Central Middlesex Hospital being carried out between 1965 and 1972, and those at the City Hospital, Nottingham, between mid 1972 and early 1974.

Twenty-seven patients were studied, of whom 13 were men and 12 women. Their ages at operation ranged from 14 to 69 (average 40), and they had had ulcerative colitis intermittently or continuously for between three and 24 years. Eight had one or more carcinomas (fig 1).

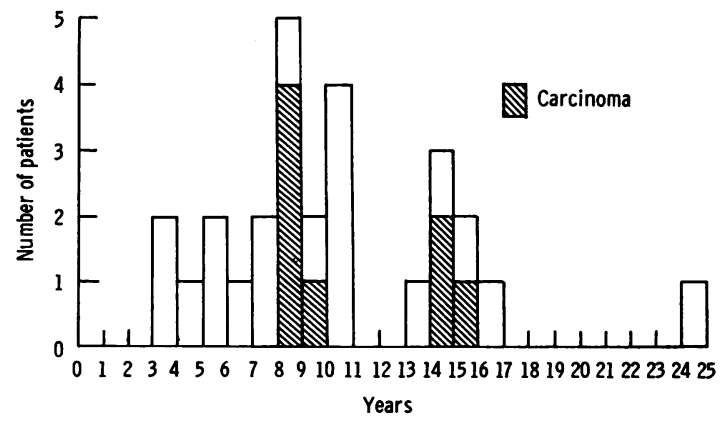

Fig 1 The duration of disease in patients with carcinoma. 
In only six cases were the gross operative specimens available for study, and the changes were those usually seen in ulcerative colitis without any specific features. Mucosal atrophy, when present, was confined to the sigmoid colon in those specimens. All the slides in each case were examined. In some instances deeper sections were cut from the blocks in order to demonstrate continuity with the mucosal

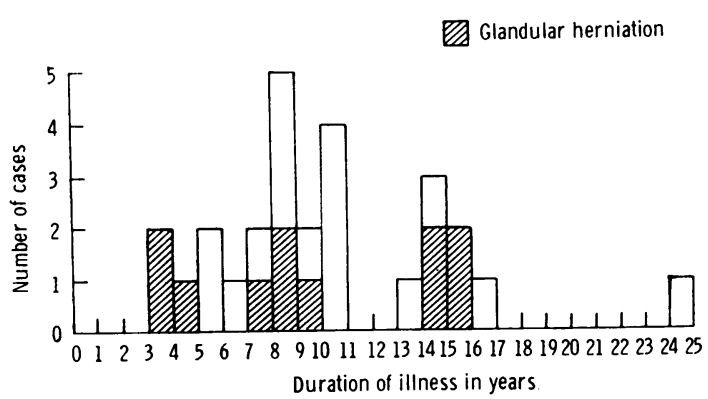

Fig 2 The duration of disease in patients with glandular herniation.

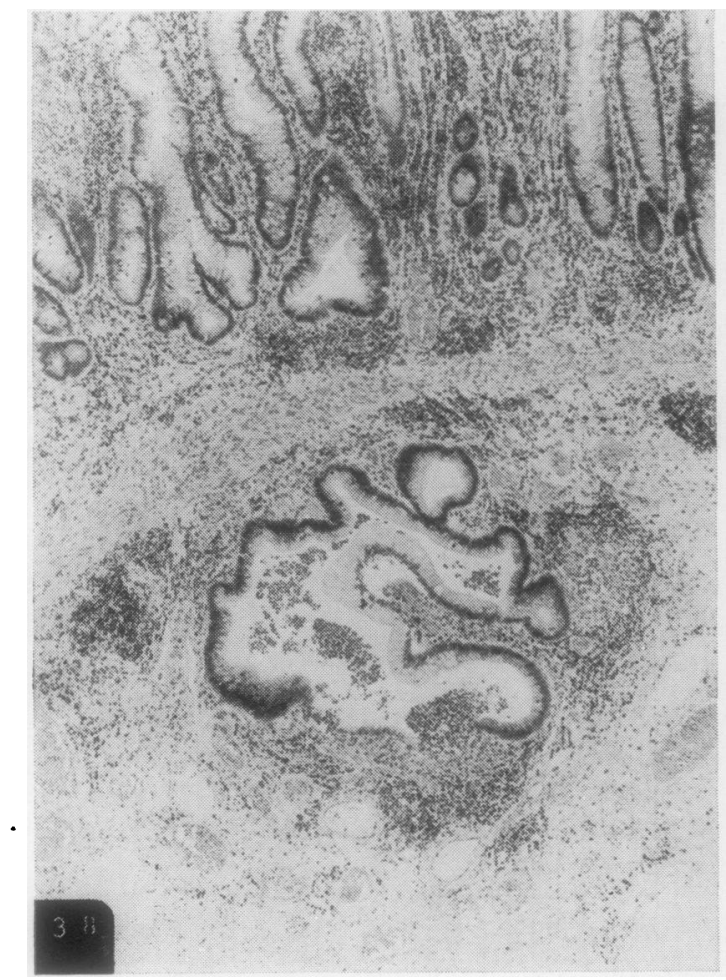

Fig 3 glands of apparently isolated islands of glandular epithelium in the submucosa.

\section{Results}

Eleven out of the 27 cases showed the presence of glandular epithelium in the submucosa forming crypts, cysts, or the walls of abscesses (fig 4), and in eight of the 11 cases misplaced epithelium was seen in more than one section, or in different areas in the same section. The misplaced epithelium appeared either as islands in the submucosa (fig 3 ) or as downgrowths of epithelium in continuity with the glands of the mucosa. Glandular invaginations and islands in the submucosa were found in sections taken from all parts of the colon. In two cases subserial sections were cut which showed that the apparent islands were not detached from the surface epithelium but were in continuity with it in a different plane from that of the original section.

\section{SITE}

In cases where resection was carried out at the time of acute exacerbation of the illness, the very severe diffuse acute and chronic inflammation made it impossible to determine the site of mucosal invagination (fig 4). But in cases where operation was carried

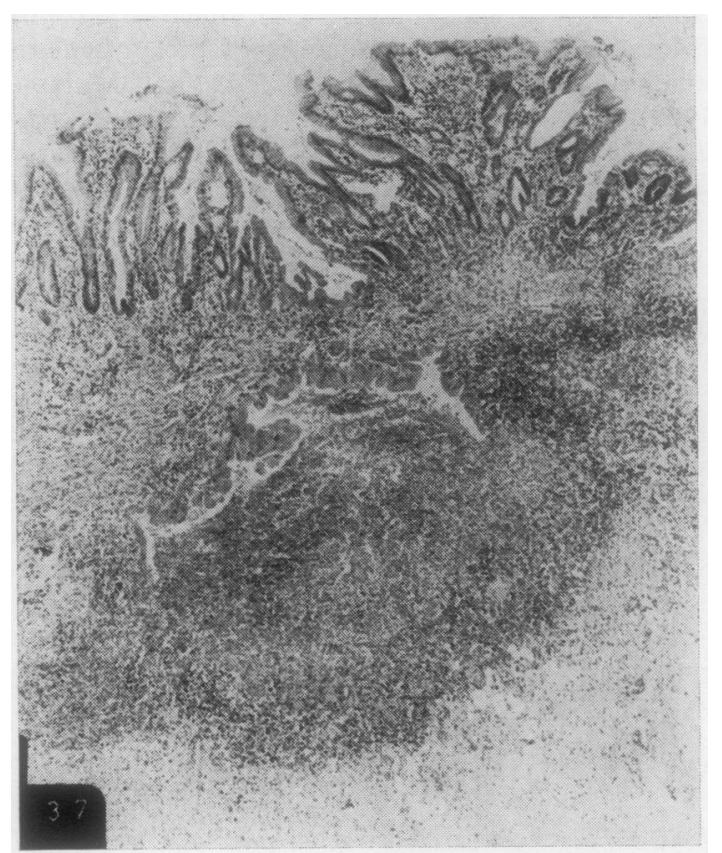

Fig 4

Fig 3 An island of mucosal glands in the submucosa. Haematoxylin and eosin $\times 56$.

Fig 4 A submucosal island of glandular epithelium partially destroyed by an abscess. Haematoxylin and eosin $\times 56$. 


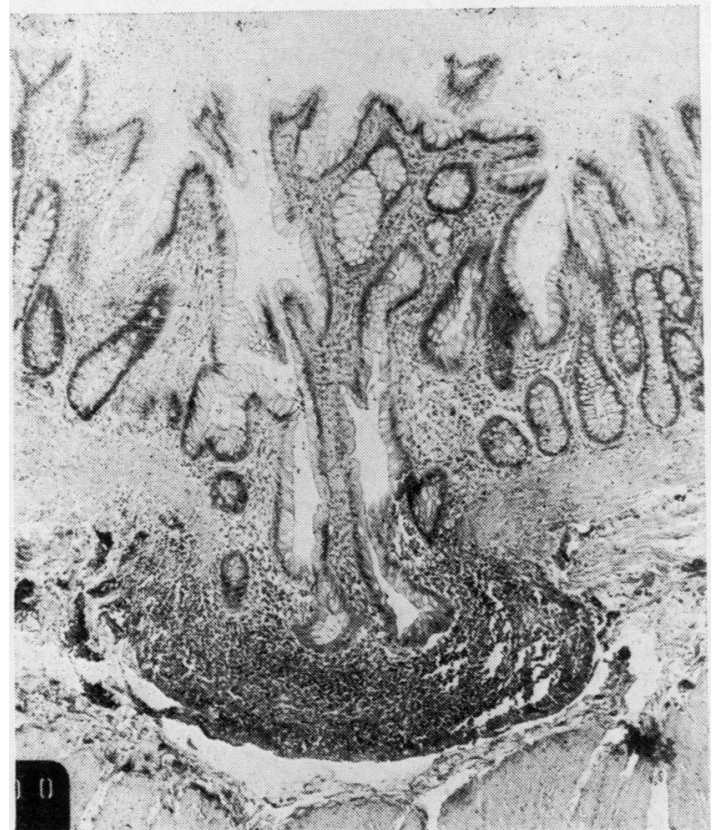

Fig 5

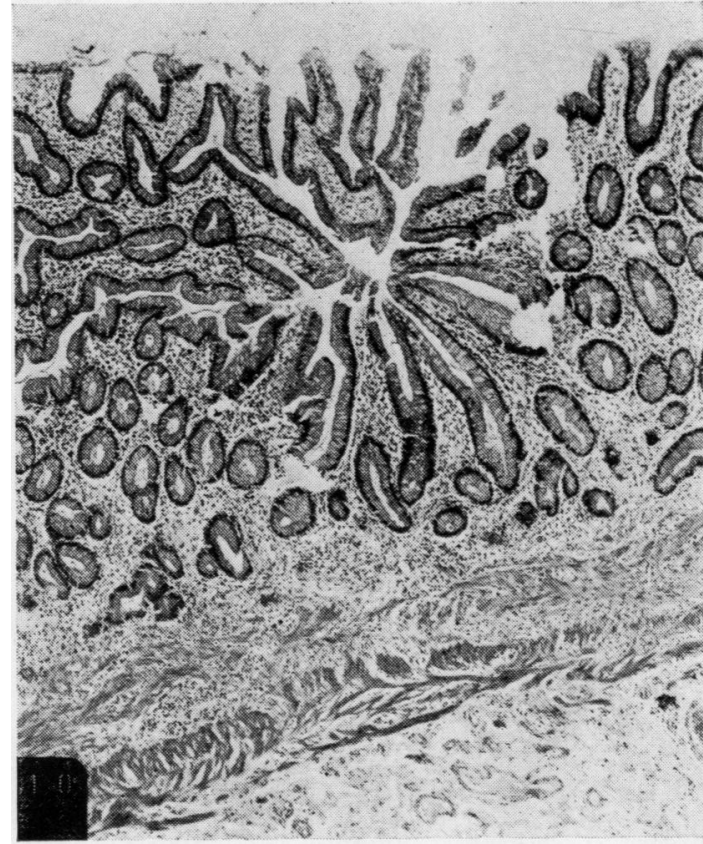

Fig 6

Fig 5 Glandular herniation extending into the submucosa. Haematoxylin and eosin $\times 56$.

Fig 6 Glandular crowding showing the 'starfish' pattern of projections into spaces. Haematoxylin and eosin $\times 56$.

out when the ulcerative process was quiescent it was clear that mucosal downgrowth or herniation through the muscularis mucosae usually took place at a point where the muscularis mucosae had been breached and replaced by a lymphoid aggregate.

Very mild degrees of glandular projection into lymphoid aggregates were observed in nearly all 27 cases studied, but projections which entered the submucosa were much less common (fig 5).

\section{Associated Factors}

\section{ASSOCIATED FACTORS}

Widening of the muscularis mucosae

Histological widening of the muscularis mucosae was present in a significant degree in eight of the 11 patients with mucosal herniation, and in six patients with no glandular herniation. It was commoner in those with longstanding disease.

\section{Glandular crowding}

The glands of the mucosa in some areas showed branching and irregularity. A characteristic finding was crowding and branching of the glands with projection into spaces, forming a 'starfish' pattern with the glands radiating from a central core (fig 6).
It has been characterized by the neutral term 'glandular crowding', rather than hyperplasia, since the appearance can be accounted for by contraction of the colon.

In nine of the cases studied there was a marked degree of glandular crowding at some point in the colon. Five of them were associated with glandular herniation (fig 7).

By contrast, mucosal atrophy, which is a common consequence of a previous attack of ulcerative colitis (Morson, 1968), was associated with glandular herniation in only one of the cases studied. Since it is characteristic of mucosal atrophy that the glands stop well short of the muscularis mucosae, this was not surprising. Mucosal atrophy was an inconstant feature in this study. It was very common in the sigmoid colon and rectum, but much rarer on the right side of the colon, and in only one case was there significant atrophy in .sections representing all parts of the colon.

In the remaining five cases with glandular herniation, the mucosal changes were variable and did not amount to either glandular crowding or atrophy.

The relationship with ulceration

Glandular herniation did not bear any relation to the 


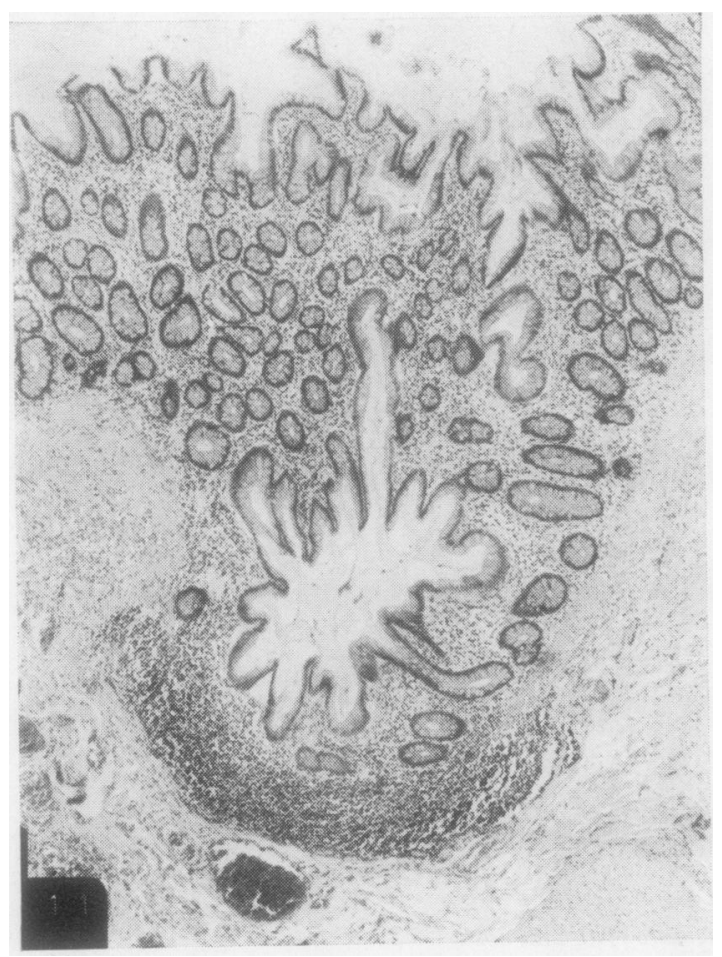

Fig 7 Glandular crowding and herniation.

Haematoxylin and eosin $\times 56$.

degree of acute inflammation at the time of operation, although the condition was obviously harder to assess if deep abscesses had been formed with the destruction of the epithelial invaginations.

The relationship to the severity of past ulceration was less easy to determine. In some of the cases studied the submucosa was obliterated and the muscularis mucosae was thick, irregular, and branching, with occasional mucosal glands among its fibres. It is probable that these cases represented the healed phases of a previous episode of severe ulcerative colitis with destruction of large parts of muscularis mucosae. But the appearance produced was quite different from that of the mucosal herniation illustrated above. There, the submucosa was an intact strip of loose connective tissue between the muscularis mucosae and the muscularis propria, and the muscularis mucosae, although usually thicker than in the normal colon, was a discrete and regular band showing no evidence of previous damage or disordered regeneration.

\section{ASSOCIATION WITH CARCINOMA}

Of the 11 patients with clear-cut glandular herniation, four had had chronic ulcerative colitis for less than eight years, whereas none of the patients with carcinoma developed malignancy within that time (fig 2). Eight patients subsequently developed carcinoma and four of them showed some degree of glandular herniation. But there were two other patients who had had ulcerative colitis for 14 and 15 years, and had extensive glandular herniation but did not develop carcinoma.

Histological evaluation of those cases with both carcinomas and glandular herniation gave no support to the view that the herniated glands were a site for potential carcinoma. Several of the carcinomas were flat and poorly differentiated, but they appeared to be in continuity with the surface epithelium, and undermining of normal mucosa by carcinoma, when it occurred, was no more than that seen in ordinary colonic cancers. None of the carcinomas had the histological appearance of having arisen in buried submucosal epithelium.

There were three cases in which there were both glandular herniations and multiple carcinomas. In one of them there were five polypoid carcinomas; the intervening epithelium of the surface and of the glandular herniations showed no significant dysplasia. In the other two, there were very extensive dysplastic and frankly malignant changes in the epithelium between the multiple invasive carcinomas but dysplasia was no more marked in the glandular herniations than on the surface.

In short, these findings suggest that glandular herniation and carcinoma are both consequences of chronic ulcerative colitis, but that they occur independently of each other and there is no causal link between them.

\section{Discussion}

This study has shown that glandular herniation or downgrowth into the submucosa in chronic ulcerative colitis is common. The condition was described by Dukes (1954) as 'misplacement of epithelium'. He believed that it was the result of deep ulceration with regeneration of the muscularis mucosae leading to isolation of glandular islands, and also that they predisposed to the development of carcinoma Displaced glandular epithelium was also mentioned by Lumb (1961) in his survey of the pathology of ulcerative colitis.

Glandular herniation such as is seen in chronic ulcerative colitis has been discussed mainly in relation to the pathogenesis of colitis cystica profunda $-a$ rare condition in which there are large mucus-filled cysts in the submucosa of the colon giving rise to clinical and radiological suspicion of carcinoma. One of the two cases of colitis cystica profunda described by Goodall and Sinclair (1957) 
occurred in a patient with chronic ulcerative colitis, and they illustrated mucosal downgrowth in that case as a possible stage in development, believing the mucosal downgrowth to arise from epithelialization of follicular ulcers which penetrated into the submucosa. Epstein, Ascari, Ablow, Seaman, and Lattes (1966) described a further four cases of colitis cystica profunda, and discussed the pathogenesis. They commented that many cases of chronic ulcerative colitis showed sequestration of glandular epithelium in the submucosa, or epithelialization of crypt abscesses, but they doubted the relationship of colitis cystica profunda to chronic inflammatory bowel disease. Wayte and Helwig, in their discussion of colitis cystica profunda (1967), distinguished between a diffuse form, which was associated with chronic inflammatory bowel disease in three of their five cases, and a focal form, where their 19 cases showed no such association.

In 1969 Clark discussed a case of chronic granulomatous disease of the colon in which 'microdiverticula' of the glandular part of the mucosa into the submucosa were a prominent feature. He subsequently (1970) described several cases referred to him, predominantly in ulcerative colitis. It was his belief that 'microdiverticula' were the initiating defect in chronic inflammatory bowel disease, being the site of crypt abscess formation and leading to the full-blown picture of ulcerative or granulomatous colitis. He believed that the initial defects in the muscularis mucosae were congenital in nature.

Thus two principal theories of origin for mucosal downgrowths or herniation in chronic ulcerative colitis emerge: that they result from epithelialization of deep abscesses, or that they are a congenital anomaly which initiate some forms of inflammatory bowel disease.

But it is not necessary to postulate ulcerative destruction or congenital defect in the muscularis mucosae to explain all forms of mucosal invagination into the submucosa. It has recently been shown that glandular epithelium may be invaginated into the submucosa in adenomatous polyps. (Muto, Bussey, and Morson, 1973; Greene, 1974). In these cases it is believed that mechanical forces in the colon are responsible.

Contraction of the muscle of the colon was put forward by Lennard-Jones, Lockhart-Mummery, and Morson (1968) as being responsible for the characteristic shortening of the colon in chronic ulcerative colitis, and in their study Goulston and McGovern (1969) demonstrated that there was chronic contraction of the muscularis mucosae, represented histologically by apparent thickening. Pressure studies in patients with ulcerative colitis have shown lower than normal pressures and an absence of phasic activity (Kern, Almy, Abbot, and Bogdonoff, 1951) interpreted as sustained but submaximal contraction.

Taken together, these findings suggest a probable mode of development of glandular herniation in chronic ulcerative colitis. This study has drawn attention to the frequency with which glandular herniations take place through the lymphoid aggregates of the muscularis mucosae, especially apparent in the quiescent cases of chronic ulcerative colitis, when they appear as focal and discrete entities. It has been found that common associations of glandular herniation are thickening of the muscularis mucosae and glandular crowding in the mucosa.

The primary initiating factor in glandular herniation in chronic ulcerative colitis is likely to be the sustained contraction of the muscularis mucosae (Goulston and McGovern, 1969). This, in its turn, leads to crowding of the mucosal glands. But there are more lymphoid aggregates in chronic ulcerative colitis and more sites of potential weakness in the muscularis mucosae. The crowded glands are then squeezed through the defects by the shortened muscle and the picture described above as glandular herniation results. A ready analogy may be drawn with diverticular disease of the colon, where the initiating factor is thought (Slack, 1966) to be sustained contraction of the muscularis propria.

It has also been shown that the presence of mucosal herniation in chronic ulcerative colitis should not be taken in itself as an indication of potential malignancy. This study indicates that mucosal herniation and carcinoma are independent sequelae of chronic ulcerative colitis, and there is no reason to assume any increased malignant potential in epithelium which is found in the submucosa as a result of mechanical forces in the muscularis mucosae.

I wish to acknowledge my thanks to the physicians and surgeons of the Central Middlesex Hospital, London, and the City Hospital, Nottingham, who have given me permission to study their patients, and to Dr Lovell of the Central Middlesex Hospital for continuing access to histological material. I am also most grateful to the Photographic Department of the General Hospital, Nottingham, and in particular to $\mathrm{Mr}$ E. McLeod for help with the photographs and diagrams.

\section{References}

Bargen, J. A., and Weber, H. M. (1931). Clinical and roentgenologic aspects of chronic ulcerative colitis. Radiology, 17, 1153-1157.

Clark, R. M. (1969). Microdiverticula: a possible cause of granulomatous ileocolitis. Canad. med. Ass. J., 100, 1025-1031.

Clark, R. M. (1970). Microdiverticula and submucosal epithelial elements in ulcerative and granulomatous diseases of the ileum and colon. Canad. med. Ass. J., 103, 24-28. 
Dukes, C. E. (1954). The surgical pathology of ulcerative colitis. Ann. roy. Coll. Surg. Engl., 14, 389-400.

Epstein, S. E., Ascari, W. Q., Ablow, R. C., Seaman, W. B., and Lattes, R. (1966). Colitis cystica profunda. Amer. J. clin. Path., 45, 186-201.

Goodall, H. B., and Sinclair, I. S. R. (1957) Colitis cystica profunda. J. Path. Bact., 73, 33-42.

Goulston, S. J. M., and McGovern, V. J. (1969). The nature of benign strictures in ulcerative colitis. New Engl. J. Med., 281, 290-295.

Greene, F. L. (1974). Epithelial misplacement in adenomatous polyps of the colon and rectum. Cancer (Philad.), 33, 206-217

Hinton, J. M. (1966). The risk of malignant change in ulcerative colitis. Gut, 7, 427-432.

Kern, F., Jr., Almy, T. P., Abbot, F. K., and Bogdonoff, M. D. (1951). The motility of the distal colon in non-specific ulcerative colitis. Gastroenterology, 19, 492-503.

Lennard-Jones, J. E., Lockhart-Mummery, H. E., and Morson, B. C. (1958). Clinical and pathological differentiation of Crohn's disease and proctocolitis. Gastroenterology, 54, 1162-1170.

Lumb, G. (1961). Pathology of ulcerative colitis. Gastroenterology, 40, 290-298.

Morson, B. C. (1968). In Ulcerative Colitis, edited by J. C. Goligher, F. T. De Dombal, J. McK. Watts, and G. Watkinson, pp. 5-31. Baillière, Tindall, and Cassell, London.

Morson, B. C. (1969). Muscle abnormality in ulcerative colitis. New Engl. J. Med., 281, 325-326.

Muto, T., Bussey, H. J. R., and Morson, B. C. (1973). Pseudo-carcinomatous invasion in adenomatous polyps of the colon and rectum. J. clin. Path., 26, 25-31.

Slack, W. W. (1966). Bowel muscle in diverticular disease. Gut, 7, 668-670.

Watts, J. McK., De Dombal, F. T., Watkinson, G., and Goligher, J. C. (1966). Long term prognosis of ulcerative colitis. Brit. med. J., 1, 1447-1453.

Wayte, D. M., and Helwig, E. B. (1967). Colitis cystica profunda. Amer. J. clin. Path., 48, 159-169. 